\title{
Evaluation of the Relationship between Janus Kinase 2 Mutational Burden and Clinical Findings in Adult Myeloproliferative Neoplasm Patients
}

\author{
- Cigdem Yuce Kahraman*, (0) Gulden Sincan** , ๑ Abdulgani Tatar* \\ ${ }^{*}$ Ataturk University Faculty of Medicine, Department of Medical Genetics, Erzurum, Turkey \\ **Ataturk University Faculty of Medicine, Department of Hematology, Erzurum, Turkey
}

Abstract

\begin{abstract}
Aim: Philadelphia-negative chronic myeloproliferative neoplasms (Ph-negative MPNs) are associated with various genetic abnormalities. The JAK2 V617F mutation is the most common one and plays a crucial role in diagnosis. We aimed to evaluate the relationship between JAK2 mutational burden and clinical parameters of MPN patients.

Methods: The present cross-sectional study was conducted on patients with MPN referred to our clinic for JAK2 mutation screening between January 2019 and December 2020. The clinical information of the patients was obtained from the hospital automation system and records in the hematology and medical genetics departments. We evaluated 143 JAK2 positive patients diagnosed with polycythemia vera, primary myelofibrosis (PMF) and essential thrombocythemia.

Results: The mean age of the patients was 60.29 (standard deviation:14.81). The mutational burden was correlated with spleen size and lactate dehydrogenase (LDH) level, particularly in PMF $(p=0.002, p=0.003$, respectively). There was no significant difference in age, gender, mutation burden and laboratory findings in patients with and without thrombosis and bleeding.

Conclusion: Clinical parameters and JAK2 mutational burden are related, but this relationship differs based on the MPN types. The spleen size in MPN, particularly massive splenomegaly and high LDH levels, may be correlated with the JAK2 mutational burden. This relationship is more pronounced for PMF. There is no significant relationship between JAK2 mutational burden and vascular complications such as thrombosis and bleeding
\end{abstract}

Keywords: Myeloproliferative disorders, Janus kinaz 2, primary polycythemia, essential thrombocythemia, primary myelofibrosis

\section{Introduction}

Philadelphia-negative chronic myeloproliferative neoplasms (Ph-negative MPNs) consist mainly of polycythemia vera (PV), primary myelofibrosis (PMF) and essential thrombocythemia (ET) (1). Ph-negative MPNs are associated with various genetic abnormalities. One of these abnormalities is Janus kinase 2 (JAK2), a protein tyrosine kinase gene. It is associated with cellular growth and proliferation. The most common genetic mutation found in Ph-negative MPNs is the JAK2 V617F mutation, wherein phenylalanine replaces valine due to a point mutation in Codon 617. The JAK2 gene is located on the short arm of chromosome 9. This mutation increases tyrosine phosphorylation activity, resulting in hypersensitivity of hematopoietic progenitor cells to growth factors $(2,3)$. Treatment options for MPN patients include acetylsalicylic acid (ASA), phlebotomy, and hydroxyurea. Where hydroxyurea cannot be used due to its side effects, interferon-alpha (IFN- $\alpha$ ), JAK2 inhibitors (ruxolitinib) or busulfan are recommended (4). JAK2 inhibitors such as ruxolitinib are more effective for splenomegaly and clinical symptoms than clonal effects. Studies on target molecules except JAK2 are ongoing (5).

The JAK2 V617F mutation occurs in approximately half of ET and PMF patients and in $90 \%$ of PV patients. Other common genetic abnormalities in MPNs include mutations in CALR and MPL. JAK2, CALR and MPL mutations have been listed under World Health Organization's MPN diagnostic criteria (6). With the NGS (next-generation 
sequencing) technique, all of these mutations can be viewed at once (7). Testing for the JAK2 V617F mutation in MPN needs to be performed routinely for diagnosis as well as for prognosis. In addition, it is opined that the mutation burden may be associated with complications related to the disease (8). The clinical course of myeloproliferative diseases is known to be quite heterogeneous. It has been stated that JAK2 V617 positivity is associated with clinical heterogeneity (9-11). Thrombosis risk in MPN patients is considered to be in low- and high-risk groups. Patients over 60 years of age and/or the presence of arterial or venous vascular complications are considered to be a highrisk group in PV. JAK2 positivity for ET is also a factor for the high-risk category (12). MPN and clonal hematopoiesis are clinical conditions that may evolve into each other, and JAK2 positivity is also seen in clonal hematopoiesis. There is a correlation between the burden of JAK2 and other genomic changes and clinical severity (13). In the literature, the clinical findings of MPN patients with and without JAK2 mutations were evaluated.

In this study, we aimed to evaluate the relationship between JAK2 mutational burden and clinical parameters of the MPN patients referred to our clinic. We evaluated the laboratory findings, spleen size, thrombosis status and treatment options of the patients.

\section{Methods}

\section{Study Design}

Written informed consent was obtained from the participants. Our study complies with the principles of the Helsinki Declaration. This study was approved by the Ataturk University Clinical Research Ethic Committee (decision number: B.30.2.ATA.0.01.00/129).

The present cross-sectional study was conducted on patients with MPN referred to our clinic for JAK2 mutation screening between January 2019 and December 2020. We identified 143 (16.94\%) JAK2 positive patients among the 844 patients who were examined. The clinical information of the MPN patients was obtained from the hospital automation system and records in the hematology and medical genetics departments.

We evaluated the laboratory parameters, clinical follow-up, thrombotic complications and JAK2 mutational burden of these JAK2 positive patients.

\section{JAK2 Genetic Analysis}

DNA isolation from patient blood samples was performed using the EZ1 DNA Blood $200 \mu \mathrm{L}$ (Qiagen) kit. DNA quality was standardized through measurement with nanodrop technology. A real-time polymerase chain reaction (PCR) study was performed with $25 \mathrm{ng}$ of purified DNA. The Ipsogen JAK2 MutaQuant Kit (Qiagen) was used for the detection and quantification of the JAK2 V617F/G1849T somatic mutation in the Rotor Gene-Q PCR system. Mutation analysis was performed with PCR software.

\section{Statistical Analysis}

The statistical analysis of laboratory results and patients' data was carried out using SPSS version 20.0. Categorical data was compared using the Pearson chisquare test. To determine the distribution of continuous variables, the Kolmogorov-Smirnov and Shapiro-Wilk tests were used; normally distributed data were presented as the mean and SD, while non-normally distributed data were presented as the median and interquartile range. The Mann-Whitney $U$ test and the Kruskal-Wallis $\mathrm{H}$ test were performed for independent samples. The threshold for statistical significance was set at $p=0.05$.

\section{Results}

There were 90 (62.9\%) PV, 34 (23.8\%) ET and 19 (13.3\%) PMF patients among a total of 143 patients. Demographic features were presented in Table 1.

When laboratory findings were evaluated according to the diagnosis, white blood cell (WBC), red blood cell $(\mathrm{RBC})$, hemoglobin ( $\mathrm{Hgb})$, hematocrit (Htc), neutrophil (neu), erythropoietin and JAK2 mutational burden showed

\begin{tabular}{|c|c|c|c|c|c|}
\hline & \multicolumn{3}{|l|}{ Diagnosis } & \multirow{2}{*}{\multicolumn{2}{|c|}{ Total }} \\
\hline & PV & ET & PMF & & \\
\hline$J A K 2(1-49 \%)$ & 53 (58.9\%) & $30(88.2 \%)$ & $9(47.4 \%)$ & \multicolumn{2}{|l|}{$92(64.3 \%)$} \\
\hline JAK2 (50-100\%) & $37(41.1 \%)$ & $4(11.8 \%)$ & $10(52.6 \%)$ & \multicolumn{2}{|l|}{$51(35.7 \%)$} \\
\hline \multirow[t]{3}{*}{ Total } & $90(100 \%)$ & $34(100 \%)$ & $19(100 \%)$ & \multicolumn{2}{|l|}{$143(100 \%)$} \\
\hline & \multicolumn{3}{|l|}{ Diagnosis } & \multirow{2}{*}{ Total } & \multirow{2}{*}{ Mean age (SD) } \\
\hline & PV & ET & PMF & & \\
\hline Male & $56(62.2 \%)$ & $14(41.2 \%)$ & $13(68.4 \%)$ & $83(58 \%)$ & 59.47 (SD: 14.99) \\
\hline Female & $34(37.8 \%)$ & $20(58.8 \%)$ & $6(31.6 \%)$ & $60(42 \%)$ & 60.37 (SD: 15.78) \\
\hline Total & $90(100 \%)$ & $34(100 \%)$ & $19(100 \%)$ & $143(100 \%)$ & 60.29 (SD: 14.81) \\
\hline
\end{tabular}


significant differences between groups (Table 2). The JAK2 mutational burden was significantly different in terms of diagnosis $(p<0.05)$ (Figure 1$)$.

Based on JAK2 mutational burden, two groups were formed: $\geq 50 \%$ and $<50 \%$. When laboratory findings were evaluated based on these groups, WBC, Hgb, neu, platelet (plt), and lactate dehydrogenase (LDH) values showed significant differences between groups. WBC, neu, LDH levels (Figure 2), spleen size (Figure 3) and median age were higher in the high mutational burden group $(p<0.05)$. Gender was not a significant factor ( $p>0.05$ ) (Table 3 ). In addition, we compared the laboratory parameters according to JAK2 mutational burden in three different MPN diagnosis groups. However, when divided into subgroups, the number of samples was unevenly insufficient and significant results could

\begin{tabular}{|c|c|c|c|c|}
\hline & PV & ET & MF & p-value \\
\hline & $\begin{array}{l}\text { Median } \\
\text { (IQR) }\end{array}$ & $\begin{array}{l}\text { Median } \\
\text { (IQR) }\end{array}$ & $\begin{array}{l}\text { Median } \\
\text { (IQR) }\end{array}$ & \\
\hline $\mathrm{Hgb}(\mathrm{g} / \mathrm{dL})$ & \begin{tabular}{|l|}
16.10 \\
$(3.67)$ \\
\end{tabular} & $15(3.38)$ & $\begin{array}{l}13.90 \\
(9.30) \\
\end{array}$ & $0.000^{*}$ \\
\hline Htc $(\%)$ & $\begin{array}{l}50.15 \\
(11.38)\end{array}$ & $\begin{array}{l}44.65 \\
(10.53)\end{array}$ & $\begin{array}{l}38.40 \\
(4.8)\end{array}$ & $0.000^{*}$ \\
\hline $\mathrm{RBC}\left(\mathrm{x} 10^{12} / \mathrm{L}\right)$ & \begin{tabular}{|l|}
6.26 \\
$(2.19)$
\end{tabular} & $5.32(1.49)$ & $\begin{array}{l}4.60 \\
(0.95)\end{array}$ & $0.000^{*}$ \\
\hline WBC $\left(\times 10^{9} / L\right)$ & $\begin{array}{l}12.44 \\
(8.70)\end{array}$ & $\begin{array}{l}10.15 \\
(3.38)\end{array}$ & $\begin{array}{l}13.90 \\
(9.30)\end{array}$ & $0.018^{*}$ \\
\hline Neu (x109/L) & $\begin{array}{l}9.06 \\
(7.88)\end{array}$ & $7.08(3.08)$ & $\begin{array}{l}9.69 \\
(9.86)\end{array}$ & $0.002^{*}$ \\
\hline PIt (x109/L) & $\begin{array}{l}570 \\
(452.25)\end{array}$ & $641(242)$ & $288(354)$ & $0.000^{*}$ \\
\hline Lymp (x109/L) & $\begin{array}{l}2.01 \\
(1.23) \\
\end{array}$ & $2.25(0.85)$ & $\begin{array}{l}1.70 \\
(0.94)\end{array}$ & 0.068 \\
\hline Uric asid (mg/dL) & $\begin{array}{l}6.10 \\
(2.30)\end{array}$ & $5.60(2.08)$ & $\begin{array}{l}5.75 \\
(3.18)\end{array}$ & 0.159 \\
\hline LDH (U/L) & $350(207)$ & $286(153)$ & $461(352)$ & 0.248 \\
\hline $\begin{array}{l}\text { Erythropoietin } \\
(\mathrm{mU} / \mathrm{mL})\end{array}$ & \begin{tabular}{|l|}
3.03 \\
$(3.65)$ \\
\end{tabular} & $4.89(2.83)$ & $\begin{array}{l}20.31 \\
(36.20)\end{array}$ & $0.000^{*}$ \\
\hline JAK2 $\%$ & $33.50(35)$ & $22.50(18)$ & $50(45)$ & $0.000^{*}$ \\
\hline Spleen size (mm) & $136.5(51)$ & $140(51)$ & $\begin{array}{l}184.5 \\
(80)\end{array}$ & $0.009 *$ \\
\hline \multirow[t]{2}{*}{ Age } & $64(24)$ & $61(23)$ & $65(13)$ & 0.473 \\
\hline & $\mathbf{N}(\%)$ & $\mathbf{N}(\%)$ & $\mathbf{N}(\%)$ & Total \\
\hline Patients & $\begin{array}{l}90 \\
(62.9 \%) \\
\end{array}$ & $34(23.8 \%)$ & $\begin{array}{l}19 \\
(13.3 \%)\end{array}$ & $\begin{array}{l}143 \\
(100 \%)\end{array}$ \\
\hline Female & \begin{tabular}{|l|}
34 \\
$(37.8 \%)$ \\
\end{tabular} & $20(58.8 \%)$ & $6(31.6 \%)$ & $\begin{array}{l}60 \\
(42 \%) \\
\end{array}$ \\
\hline Male & $\begin{array}{l}56 \\
(62.2 \%)\end{array}$ & $14(41.2 \%)$ & $\begin{array}{l}13 \\
(68.4 \%)\end{array}$ & $\begin{array}{l}83 \\
(58 \%)\end{array}$ \\
\hline \multicolumn{5}{|c|}{$\begin{array}{l}\text { Htc: Hhematocrit, Hgb: Hemoglobin, RBC: Red blood cell, WBC: White blood cell, } \\
\text { neu: Neutrophilia, plt: Platelet, lymp: Lymphocyte, LDH: Lactate dehydrogenase, } \\
\text { IQR: Interquartile range } \\
\text { *significant p-values, detected by the Kruskal-Wallis test }\end{array}$} \\
\hline
\end{tabular}

not be obtained. Significant differences in WBC, Hgb, neu, LDH, uric acid, and spleen size were detected only in the PV group because of the relatively higher sample number $(p<0.05)$. Thrombosis occurred in $26(18.2 \%)$ patients out of 143 , while gastrointestinal bleeding occurred in three $(2.1 \%)$ patients. Among the patients with thrombosis, four had cerebrovascular events, two had pulmonary thromboembolism, ten had myocardial infarction, four had pulmonary vein thrombosis, two had splenic vein thrombosis, one had deep vein thrombosis and one had digital arterial thrombosis. When the groups with and without thrombosis were compared, there was

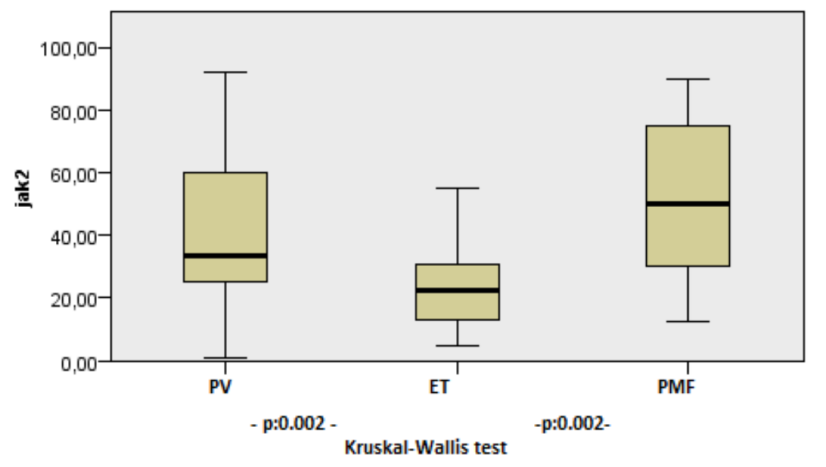

Figure 1. JAK2 mutational burden in terms of diagnosis PMF: Primary myelofibrosis, PV: Polycythemia vera, ET: Essential thrombocythemia, JAK2: Janus kinase 2

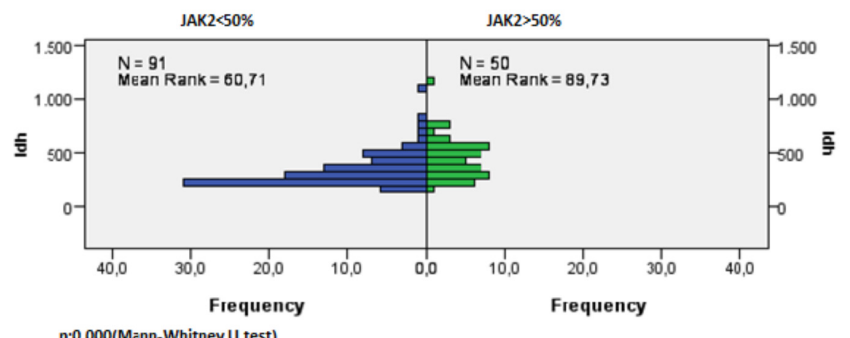

p:0.000(Menn-Whitney U test)

Figure 2. LDH levels in terms of JAK2 mutational burden JAK2: Janus kinase 2, LDH: Lactate dehydrogenase

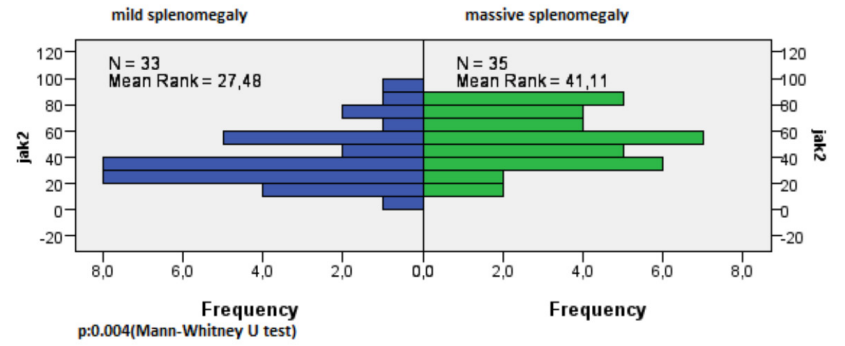

Figure 3. JAK2 mutational burden in terms of splenomegaly category

JAK2: Janus kinase 2 
no statistical difference in age, JAK2 mutational burden, spleen size and laboratory findings. Only the lymphocyte count was lower $(p<0.05)$. Gender was not a significant factor ( $p>0.05)$. Eighteen PV, seven ET patients, and one PMF patient were part of the thrombosis group.

Cases with a spleen size $\geq 160 \mathrm{~mm}$ in the ultrasonography were considered as massive splenomegaly (SM) and those with a spleen size of 130-160 mm were considered as mild splenomegaly. Laboratory findings were evaluated according to mild and massive SM groups (Table 4). The SM rate was $57.1 \%$ in the group having a $J A K 2 \geq 50 \%$ mutation burden. Splenomegaly was found to be associated with JAK2 mutation burden $(p=0.002)$ (Table 2). The SM rates were $48.7 \%$ in PV, 35.3\% in ET and $83.3 \%$ in PMF patients. The PMF group was significantly different from the other groups $(p=0.009)$.

We also evaluated the treatment protocols of the patients. Most of the patients were treated with ASA+hydroxyurea $(59.4 \%) ; 64.7 \%$ of the patients using this treatment were PV patients, $22.4 \%$ were $\mathrm{ET}$, and $12.9 \%$ were PMF patients. The second most common treatment options were ASA (14.7\%) and ASA+phlebotomy (14.7\%). $71.4 \%$ of those treated with

Table 3. Laboratory findings in terms of JAK2 mutational burden

\begin{tabular}{|c|c|c|c|}
\hline & JAK2\% (1-49) & JAK2\% (50-100) & p-value \\
\hline & Median (IQR) & Median (IQR) & \\
\hline $\mathrm{Hgb}(\mathrm{g} / \mathrm{dL})$ & $15.65(3.20)$ & $13.50(4.60)$ & $0.005^{*}$ \\
\hline Htc (\%) & $49.30(9.60)$ & $47.10(16.10)$ & 0.141 \\
\hline $\mathrm{RBC}\left(\mathrm{x} 10^{12} / \mathrm{L}\right)$ & $5.82(1.63)$ & $5.15(3.11)$ & 0.677 \\
\hline WBC (x109/L) & $10.325(4.98)$ & $16.130(10.52)$ & $0.000^{*}$ \\
\hline Neu $\left(\times 10^{9} / L\right)$ & $7.26(4.28)$ & $12.54(8.87)$ & $0.000^{*}$ \\
\hline Plt (x109/L) & $644(397.5)$ & 439. (364) & $0.001^{*}$ \\
\hline $\begin{array}{l}\text { Lymp } \\
\left(\times 10^{9} / \mathrm{L}\right)\end{array}$ & $2.11(1.03)$ & $2.14(1.25)$ & 0.066 \\
\hline $\begin{array}{l}\text { Uric asid } \\
(\mathrm{mg} / \mathrm{dL})\end{array}$ & $5.72(1.84)$ & $6.76(2.50)$ & 0.3 \\
\hline LDH (U/L) & $287(159)$ & $436.5(241))$ & $0.003^{*}$ \\
\hline $\begin{array}{l}\text { Eritropoetin } \\
(\mathrm{mU} / \mathrm{mL})\end{array}$ & $4.31(4.54)$ & $3.34(4.37)$ & 0.5 \\
\hline Age & $61(22)$ & $65(16)$ & $0.003^{*}$ \\
\hline \multirow[t]{2}{*}{$\begin{array}{l}\text { Spleen size } \\
(\mathrm{mm})\end{array}$} & $133(48)$ & $162(76)$ & $0.002^{*}$ \\
\hline & N (\%) & N (\%) & \\
\hline Patients & $92(64.3 \%)$ & $51(35.7 \%)$ & \multirow{3}{*}{0.8} \\
\hline Female & $38(63.3 \%)$ & $22(36.7 \%)$ & \\
\hline Male & $54(65.1 \%)$ & $29(34.9 \%)$ & \\
\hline \multicolumn{4}{|c|}{$\begin{array}{l}\text { Htc: Hematocrit, Hgb: Hemoglobin, RBC: Red blood cell, WBC: White blood cell,, } \\
\text { neu: Neutrophil, plt: Platelet, lymp: Lymphocyte, LDH: Lactate dehydrogenase, } \\
\text { IQR: Interquartile range } \\
\text { *significant p-values, detected by the Mann-Whitney U test and means the } \\
\text { distrubution of the parameters was not the same across categories of JAK2 } \\
\text { mutational burden }\end{array}$} \\
\hline
\end{tabular}

ASA were ET patients, $23.8 \%$ were PV patients, and $4.8 \%$ were PMF patients. $100 \%$ of the patients treated with ASA+phlebotomy were PV patients. The other treatment protocols included ASA+ruxolitinib in $4.2 \%, A S A+I F N-\alpha$ in $3.5 \%$, ruxolitinib in $1.4 \%$, clopidogrel-hydroxyurea in $0.7 \%$, hydroxyurea in $0.7 \%$ and allogenic bone marrow transplantation in $0.7 \%$ of the patients. Htc and lymph levels were significantly higher in lower-aged patients treated with ASA+phlebotomy than in patients treated with ASA+hydroxyurea. The JAK2 mutational burden, mean age and LDH level were significantly higher in patients treated with ASA+hydroxyurea than in those treated with ASA alone. The patients treated with ASA+hydroxyurea were older than the patients treated with ASA and ASA+phlebotomy. Twenty-one thrombosis patients were treated with ASA+hydroxyurea, three with ASA+IFN- $\alpha$, one with ASA+ruxolitinib, and one with clopidogrel+hydroxyurea.

\section{Discussion}

Based on laboratory findings, thrombosis status, and treatments, we evaluated the JAK2 V617F positive MPN patients. There have been studies evaluating MPN patients with and without JAK2 V617F mutation in only

\begin{tabular}{|c|c|c|c|}
\hline & $\begin{array}{l}\text { Spleen size (130- } \\
159 \mathrm{~mm})\end{array}$ & $\begin{array}{l}\text { Spleen size } \\
(\geq 160 \mathrm{~mm})\end{array}$ & p-value \\
\hline & Median (IQR) & Median (IQR) & \\
\hline $\mathrm{Hgb}(\mathrm{g} / \mathrm{dL})$ & $15.30(4.05)$ & $12.60(4.30)$ & $0.003^{*}$ \\
\hline Htc (\%) & $48.40(11.90)$ & $40.20(13.20)$ & $0.006^{*}$ \\
\hline $\operatorname{RBC}\left(\times 10^{12} / \mathrm{L}\right)$ & $5.91(2.20)$ & $4.80(2.22)$ & $0.008^{*}$ \\
\hline WBC $\left(\times 10^{9} / L\right)$ & $11.02(8.14)$ & $11.46(12.43)$ & 0.830 \\
\hline neu $\left(\times 10^{9} / L\right)$ & 7.67 (7.39) & $8.80(8.38)$ & 0.480 \\
\hline Plt $\left(\times 10^{9} / \mathrm{L}\right)$ & $623(304.5)$ & 391 (336) & $0.005^{*}$ \\
\hline \begin{tabular}{|l|} 
Lymp \\
$\left(\times 10^{9} / \mathrm{L}\right)$ \\
\end{tabular} & $2.19(1.18)$ & $1.41(1.00)$ & $0.018^{*}$ \\
\hline $\begin{array}{l}\text { Uric asid } \\
(\mathrm{mg} / \mathrm{dL})\end{array}$ & $5.90(2.60)$ & $6.60(2.85)$ & 0.299 \\
\hline LDH (U/L) & $315(216)$ & $504(224.75)$ & $0.007^{*}$ \\
\hline \begin{tabular}{|l|}
$\begin{array}{l}\text { Eritropoetin } \\
(\mathrm{mU} / \mathrm{mL})\end{array}$ \\
\end{tabular} & 3.65 (3.55) & $4.04(4.31)$ & 0.716 \\
\hline JAK2 \% & $30(29)$ & $52(40)$ & $0.004^{*}$ \\
\hline Age & $64(17)$ & $64(25)$ & 0.917 \\
\hline \multirow[t]{2}{*}{$\begin{array}{l}\text { Spleen size } \\
(\mathrm{mm})\end{array}$} & $138.30(14)$ & $180(54)$ & $0.000 *$ \\
\hline & $\mathbf{N}(\%)$ & N (\%) & \\
\hline Patients & $33(48.53 \%)$ & $35(51.47 \%)$ & \\
\hline \multicolumn{4}{|c|}{$\begin{array}{l}\text { Htc: Hematocrit, Hgb: Hemoglobin, RBC: Red blood cell, WBC: White blood cell, } \\
\text { neu: Neutrophil, plt: Platelet, lymp: Lymphocyte, LDH: Lactate dehydrogenase, } \\
\text { IQR: Interquartile range } \\
\text { *significant p-values, detected by the Mann-Whitney U test and means the } \\
\text { distrubution of the parameters was not the same across categories of spleen size }\end{array}$} \\
\hline
\end{tabular}


PV (11), in only ET $(9,14-17)$, both in PV and ET (18-20), in ET and PMF (1), and in PV, ET and PMF (21). In these studies, within the JAK2 positive group, $\mathrm{Hgb}(18,22)$, Htc $(1,14,15,19)$, RBC $(20)$ levels were significantly higher, and plt $(1,14,15,18,20)$ levels were significantly lower. In various other studies, WBC $(1,18-20,22)$ and neu (9) were also significantly higher. In our study, we evaluated PV, ET and PMF patients who already had the JAK2 mutation.

There are studies evaluating the JAK2 mutational burden in MPN patients, which are similar to our study. In a study by Tefferi et al. (11), JAK2 heterozygous and homozygous PV patients were compared, and no statistical difference was found in age, gender, leukocyte or platelet counts, or thrombosis or bleeding cases, at the time of diagnosis. However, in homozygous patients, a significantly higher hemoglobin level at the time of diagnosis, increased pruritus, and a higher rate of fibrotic transformation were found (11). In the study by Zhou et al. (20), the JAK2 V617F mutational burden was found to be significantly higher in PV compared to ET and PMF patients, similar to our study. It was reported that the JAK2 mutational burden was positively correlated with WBC and plt counts in ET patients, and with WBC and RBC counts in PV patients (20). In the study by Liu et al. (21), high to low JAK2 mutation burden was determined in patients with PV, PMF and ET, respectively. Patient age and $W B C$ in $P V, W B C$ in ET, and Hgb, WBC, and plt count in PMF were significantly correlated with higher mutation burden (21). In our study, Htc, Hgb, and RBC were significantly higher in the PV group compared to the ET and PMF groups, which is consistent with previous studies $(18,20,23)$; while WBC, neu were significantly higher in the PV group compared to the ET group. In contrast to our study, Vannucchi et al. (19), indicated that age, Htc, and WBC levels were not different in terms of the diagnosis of PV or ET. In some studies, LDH levels were not different in JAK2 mutant patients $(1,9,17,24)$, while in other studies, LDH was different, consistent with our study (25).

In the study by Kittur et al. (14), it was stated that JAK2 mutational burden was associated with splenomegaly and male gender in ET patients. Several studies have found a link between splenomegaly and JAK2 mutations in ET patients $(1,15,18,19)$. In our study, SM and JAK2 mutational burden were related, but gender was not related, as in various other studies $(16,26)$. According to our study, patients with SM may have a higher JAK2 mutational burden.

There is conflicting information in the literature about the relationship between the JAK2 mutation and thrombosis. Several studies have found that JAK2 mutations are linked to thrombosis in MPN patients $(9,14,16,19,27,28)$. According to another study, MPN patients with an allele burden of $\geq 50 \%$ JAK2 patients had an increased risk of vascular complications, and they account for the majority of PV and a small proportion of ET (27). Thrombosis or bleeding risk, on the other hand, was not found to be associated with the JAK2 mutation in myeloproliferative diseases in various studies $(1,11,17,22,29)$. In our study, there was no significant difference between the groups with and without thrombosis, in terms of age, gender, JAK2 mutational burden and laboratory parameters except lymph count. The lymph count was significantly higher in the group without thrombosis. This result may be due to the small number of patients with thrombosis. Across studies, low/high-risk of thrombosis groups are defined for PV (30) and ET patients $(12,30)$. ASA is recommended in low-risk patients to prevent venous and arterial thrombosis. Phlebotomy is performed in both high and low-risk groups to keep the hematocrit values below $45 \%$ (12). Low-dose ASA, phlebotomy and hydroxyurea are recommended for patients in the high-risk category. Hydroxyurea regulates the plt count and reduces the risk of vascular complications. IFN- $\alpha, J A K 2$ inhibitor or Busulfan are used when hydroxyurea cannot be used due to its side effects (4). In our study, consistent with the literature, ASA+hydroxyurea, ASA+phlebotomy and ASA were the common treatment options and early preventive treatments may be protective for thrombosis.

\section{Study Limitations}

Although our total number of patients was sufficient, when we evaluated the three different groups of MPNs, the number of samples was low in the ET and PMF groups, in particular. When the subgroups were classified according to JAK2 mutational burden, a strong comparison could not be made because the numbers were further reduced. This is the limited aspect of our study. Despite these limitations, there have been few articles on the effects of JAK2 mutational burden in all three MPNs, and we believe that this article will contribute to the literature.

\section{Conclusion}

Clinical parameters and JAK2 mutational burden are related but this relationship differs based on the MPN types. The spleen size in MPN, particularly SM and high LDH level may be correlated with the JAK2 mutational burden. This relationship is more pronounced for PMF. There is no significant relationship between JAK2 mutational burden and vascular complications such as thrombosis and bleeding.

\section{Ethics}

Ethics Committee Approval: This study was approved by Ataturk University Clinical Research Ethic Committee (decision number: B.30.2.ATA.0.01.00/129). 
Informed Consent: Retrospective study.

\section{Authorship Contributions}

Concept: C.Y.K., G.S., A.T., Design: C.Y.K., G.S., A.T., Data Collection or Processing: C.Y.K., G.S., Analysis or Interpretation: C.Y.K., G.S., A.T., Literature Research: C.Y.K., G.S., Writing: C.Y.K., G.S.

Conflict of Interest: No conflict of interest was declared by the authors.

Financial Disclosure: The authors declared that this study received no financial support.

\section{Acknowledgements}

This manuscript was reviewed as a preprint in Research Square, a research communication platform. Research Square preprints are published under a CC-BY 4.0 license, meaning the author retains full copyright for their work. When the paper is accepted and published in a journal, a link to the final version of record can be placed on the preprint, guiding readers to the published paper (https:// www.researchsquare.com/article/rs-305849/v1).

\section{References}

1. Yönal i, Dağlar-Aday A, Akadam-Teker B, et al. Impact of JAK2V617F Mutational Status on Phenotypic Features in Essential Thrombocythemia and Primary Myelofibrosis. Turk J Haematol 2016;33:94-101.

2. Kralovics R, Passamonti F, Buser AS, et al. A gain-of-function mutation of JAK2 in myeloproliferative disorders. N Engl J Med 2005;352:1779-90.

3. Lee $\mathrm{E}$, Lee $\mathrm{KJ}$, Park $\mathrm{H}$, et al. Clinical Implications of Quantitative JAK2 V617F Analysis using Droplet Digital PCR in Myeloproliferative Neoplasms. Ann Lab Med 2018;38:147-54.

4. Hintermair S, Zwickl-Traxler E, Pecherstorfer M, et al. Evaluation of vascular events in patients with myeloproliferative syndromes and mutations of either the januskinase-2 or calreticulin gene at the university hospital Krems from 2008 to 2015. Oncotarget 2018;9:8450-62.

5. Brkic S, Meyer SC. Challenges and Perspectives for Therapeutic Targeting of Myeloproliferative Neoplasms. Hemasphere 2021;5:e516.

6. Tefferi A. Primary myelofibrosis: 2021 update on diagnosis, risk-stratification and management. Am J Hematol 2021;96:145-62.

7. Kearney L, Lee Tokar L, Flynn C, Mykytiv V, Murphy K, Langabeer SE. Repeat JAK2 V617F testing in patients with suspected essential thrombocythaemia. J Clin Pathol 2020;73:772.

8. Borowczyk M, Wojtaszewska M, Lewandowski K, et al. The JAK2 V617F mutational status and allele burden may be related with the risk of venous thromboembolic events in patients with Philadelphia-negative myeloproliferative neoplasms. Thromb Res 2015;135:272-80.

9. Campbell PJ, Scott LM, Buck G, et al. Definition of subtypes of essential thrombocythaemia and relation to polycythaemia vera based on JAK2 V617F mutation status: a prospective study. Lancet 2005;366:1945-53.

10. Nielsen C, Birgens HS, Nordestgaard BG, Bojesen SE. Diagnostic value of JAK2 V617F somatic mutation for myeloproliferative cancer in 49488 individuals from the general population. Br J Haematol 2013;160:70-9.

11. Tefferi A, Lasho TL, Schwager SM, et al. The clinical phenotype of wild-type, heterozygous, and homozygous JAK2V617F in polycythemia vera. Cancer 2006;106:631-5.

12. Tefferi A, Barbui T. Polycythemia vera and essential thrombocythemia: 2021 update on diagnosis, riskstratification and management. Am J Hematol 2020;95:1599613.

13. Moliterno AR, Kaizer $H$. Applied genomics in MPN presentation. Hematology Am Soc Hematol Educ Program 2020;2020:434-9.

14. Kittur J, Knudson RA, Lasho $T L$, et al. Clinical correlates of JAK2V617F allele burden in essential thrombocythemia. Cancer 2007;109:2279-84.

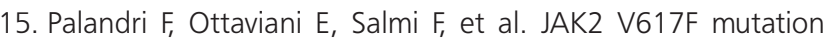
in essential thrombocythemia: correlation with clinical characteristics, response to therapy and long-term outcome in a cohort of 275 patients. Leuk Lymphoma 2009;50:247-53.

16. Bayram M, Özkocaman V, Özkalemkaş F, et al. Esansiyel Trombositoz Tanısıyla İzlenen Olgularda JAK-2 Gen Mutasyonu ve Komplikasyonlarla Ilişkisi. Uludağ Üniversitesi Tıp Fakültesi Dergisi 2011;37:13-6.

17. Antonioli E, Guglielmelli P, Poli G, et al. Influence of JAK2V617F allele burden on phenotype in essential thrombocythemia. Haematologica 2008;93:41-8.

18. Karkucak M, Yakut T, Ozkocaman V, et al. Evaluation of the JAK2-V617F gene mutation in Turkish patients with essential thrombocythemia and polycythemia vera. Mol Biol Rep 2012;39:8663-7.

19. Vannucchi AM, Antonioli E, Guglielmelli $P$, et al. Clinical profile of homozygous JAK2 617V>F mutation in patients with polycythemia vera or essential thrombocythemia. Blood 2007; 110:840-6.

20. Zhou J, Ye Y, Zeng S, et al. Impact of JAK2 V617F mutation on hemogram variation in patients with non-reactive elevated platelet counts. PLoS One 2013;8:e57856.

21. Liu Y, Liu C, He N, et al. [JAK2 V617F mutation burden and its clinical implications in 415 patients with myeloproliferative neoplasm]. Zhonghua Xue Ye Xue Za Zhi 2015;36:191-5.

22. Lieu $\mathrm{CH}$, Wu HS, Hon YC, et al. Prevalence of the JAK2-V617F mutation in Taiwanese patients with chronic myeloproliferative disorders. Intern Med J 2008;38:422-6.

23. Cetin G, Ozkan T, Turgut S, et al. Evaluation of clinical and laboratory findings with JAK2 V617F mutation as an independent variable in essential thrombocytosis. Mol Biol Rep 2014;41:6737-42. 
24. Campbell PJ, Griesshammer M, Döhner K, et al. V617F mutation in JAK2 is associated with poorer survival in idiopathic myelofibrosis. Blood 2006;107:2098-100.

25. Alvarez-Larrán A, Cervantes F, Bellosillo B, et al. Essential thrombocythemia in young individuals: frequency and risk factors for vascular events and evolution to myelofibrosis in 126 patients. Leukemia 2007;21:1218-23.

26. Cheung B, Radia D, Pantelidis P, et al. The presence of the JAK2 V617F mutation is associated with a higher haemoglobin and increased risk of thrombosis in essential thrombocythaemia. Br J Haematol 2006;132:244-5.

27. Carobbio A, Finazzi G, Antonioli E, et al. JAK2V617F allele burden and thrombosis: a direct comparison in essential thrombocythemia and polycythemia vera. Exp Hematol 2009;37:1016-21.

28. Zhang $Y$, Zhou $Y$, Wang $Y$, et al. Thrombosis among 1537 patients with JAK2(V617F) -mutated myeloproliferative neoplasms: Risk factors and development of a predictive model. Cancer Med 2020;9:2096-105.

29. Ayer M, Menken I, Yamak M, Ayer FA, Kırkızlar O, Burak Aktuğlu M. The Impact of Mean Platelet Volume (MPV) and JAK-2 Mutation on Thrombosis in Chronic Myeloproliferative Diseases. Indian J Hematol Blood Transfus 2017;33:181-7.

30. Iurlo A, Cattaneo D, Bucelli C, Baldini L. New Perspectives on Polycythemia Vera: From Diagnosis to Therapy. Int J Mol Sci 2020;21:5805. 